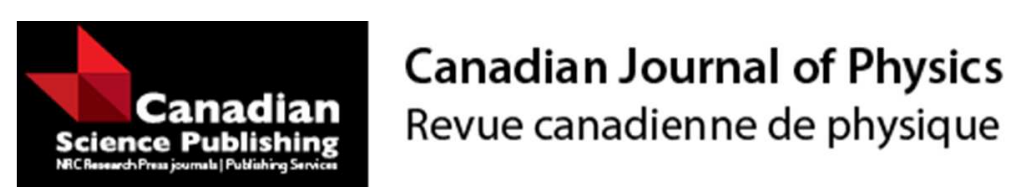

\title{
A summary of results obtained with the cryogenic electrostatic storage ring DESIREE
}

\begin{tabular}{|r|l|}
\hline Journal: & Canadian Journal of Physics \\
\hline Manuscript ID & cjp-2016-0872 \\
\hline Manuscript Type: & Article \\
\hline Date Submitted by the Author: & 26-Nov-2016 \\
\hline Complete List of Authors: & Zettergren, Henning; Stockholms Universitet, \\
\hline Keyword: & $\begin{array}{l}\text { electrostatic storage rings, atomic and molecular anions, lifetimes of } \\
\text { excited states, cluster cooling, rotational cooling }\end{array}$ \\
\hline
\end{tabular}

SCHOLARONE $^{\text {m }}$
Manuscripts 


\title{
A summary of results obtained with the cryogenic electrostatic storage ring DESIREE
}

\author{
H. Zettergren \\ Department of Physics, Stockholm University, AlbaNova University center, SE-106 91 Stockholm, Sweden
}

\begin{abstract}
:
This paper highlights results from recent studies of cryogenically cooled atomic and molecular anions carried out at the DESIREE storage ring facility at Stockholm University in Stockholm, Sweden. These results include measurements of lifetimes of excited metastable states in atomic anions $\left(\mathrm{S}^{-}, \mathrm{Se}^{-}, \mathrm{Te}^{-}, \mathrm{Ni}^{-}\right.$, and $\left.\mathrm{Pt}^{-}\right)$, pilot studies of rotational cooling of $\mathrm{OH}^{-}$, and cooling and decay of cluster anions exemplified by results for $\mathrm{Ag}_{5}^{-}$.
\end{abstract}

Keywords: Electrostatic storage rings, atomic and molecular anions, lifetimes of excited states, cluster cooling, rotational cooling.

\section{Introduction}

The development of electrostatic ion-beam storage rings has opened up a new era for studies of atomic and molecular processes in new time domains. Such ion storage devices can typically be made more compact than large-scale magnetic storage rings. Further advantages are that magnetic field mixing is avoided in lifetime measurements and that ions with high-mass-to-charge ratios may be easily stored due to the electrostatic confinement. This in combination with the development of efficient ion beam production methods (e.g. electrospray ionization [1]) have paved the way for studies of complex and fragile molecular systems. Pioneering studies on small clusters [2,3] biomolecules [4,5], PAHs [6], and fullerenes [7] have been performed earlier in room temperature storage rings in Aarhus [8], Tokyo [9], and Lyon [10]. In such experiments, the residual gas pressure (giving unwanted collisions) and the black body radiation background (unwanted photo-absorption) often limit the ion storage times to tens of seconds or less.

Recent developments of cryogenically cold ion storage rings have opened up for studies of inherent atomic and molecular properties in completely new time domains. Examples here are DESIREE in Stockholm [11,12], CSR (Cryogenic Storage Ring) in Heidelberg [13], and RICE (RIken Cryogenic E-ring) in Tokyo [14]. The cold, low density $\left(\sim 10^{-16}\right.$ $10^{-14}$ mbar physical pressure), environments in cryogenic electrostatic ion storage devices are ideal for lifetime measurement and studies of cooling processes in unprecedented details. This was first demonstrated in lifetime measurements of the metastable $\mathrm{He}^{-}\left(1 \mathrm{~s} 2 \mathrm{~s} 2 \mathrm{p}^{4} \mathrm{P}_{5 / 2}\right)$ state with the CONEtrap in Stockholm [15], and in studies of $\mathrm{SF}_{6}{ }^{-}$in the CTF Cryogenic Trap for Fast ion beams in Heidelberg [16]. More recent examples are measurements of lifetimes of excited metastable states of atomic anions in DESIREE $[17,18,19]$ and studies of photodissociation of $\mathrm{CH}^{+}$in $\mathrm{CSR}$ [20]. In this paper, we give an overview and highlight some of our recent results from studies of 
negatively charged atomic, molecular and cluster ions in DESIREE single-ring experiments. A recent review of electrostatic storage rings is given in Ref. [21].

\section{Experiment}

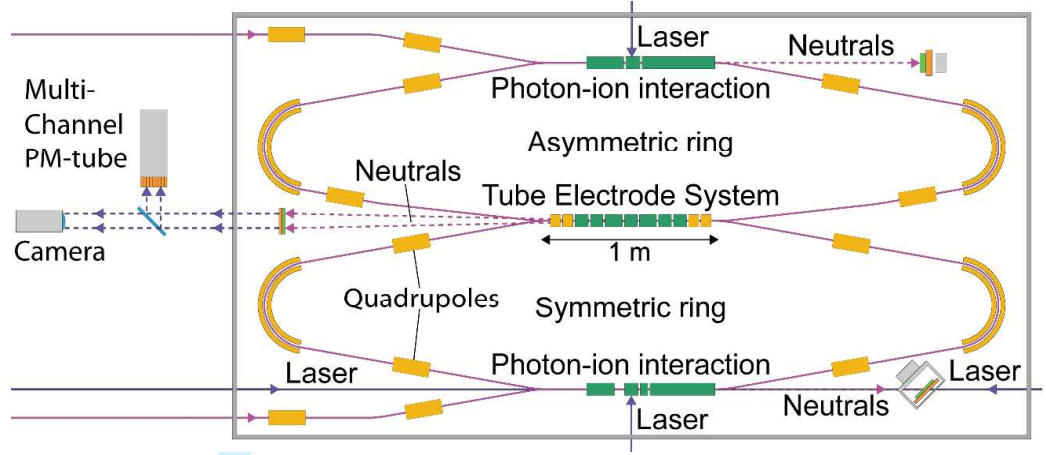

Figure 1: Schematic of the DESIREE ion-beam storage rings [11,12].

The DESIREE (Double ElectroStatic Ion Ring ExpEriment) facility at Stockholm University has two ion storage rings and a common section for studies of reactions between oppositely charged ions (see Fig. 1). Such merged-beams experiments are now possible at collision energies of hundreds of $\mathrm{eV}$, and we are currently commissioning experiments also at sub-thermal energies. DESIREE is cryogenically cooled to $13 \mathrm{~K}$ and has an extremely low residual gas background pressure $\left(\sim 10^{-14} \mathrm{mbar}\right)$. This allows for the first measurements of mutual neutralization rates with atomic and molecular systems under astrophysical conditions. The excellent experimental conditions (cold, empty, dark) are also ideal for lifetime measurements and action spectroscopy on isolated atomic or molecular ions in single ring experiments.

The single ring experiments presented in this paper were carried out using the symmetric storage ring in Fig. 1. The anions were produced in a cesium sputter ion source, accelerated to $\mathrm{keV}$ energies, and mass selected before injection and storage at these energies. When neutrals are formed from electron emission or dissociating hot molecules or clusters in one of the two straight sections of the ring, they are no longer deflected by the electrostatic ion-optical elements and follow straight-line trajectories towards detectors (see Fig. 1). The decay of the stored anions may in this way be monitored as a function of time. For atomic anions, the decay is dominated by collisions with the residual gas giving storage lifetimes of tens of minutes due to the low-density environment. This allows for studies of the spontaneous decay of long-lived metastable excited states by using lasers to state-selectively probe the populations in specific excited (metastable) states as functions of time. In contrast to atomic ions, molecular ions may store energy in rotations and vibrations. In such cases, the spontaneous decay of hot ions gives information on cooling dynamics, e.g. on vibrational autodetachment [16] and various types of photon emission processes $[2,3,6]$. Here, laser probing may be used to provide more detailed information on for instance the rotational temperature of the stored ions. 


\section{Results and discussion}

\subsection{Lifetime measurements of long-lived excited bound atomic anion states}

\begin{tabular}{|l|l|l|}
\hline Anion & Transition & Lifetime, $\tau$ \\
\hline $\mathrm{S}^{-}$ & $3 \mathrm{p}^{52} \mathrm{P}_{1 / 2} \rightarrow 3 \mathrm{p}^{52} \mathrm{P}_{3 / 2}$ & $503 \pm 54 \mathrm{~s}[17]$ \\
$\mathrm{Se}^{-}$ & $4 \mathrm{p}^{5} \mathrm{P}_{1 / 2} \rightarrow 4 \mathrm{p}^{52} \mathrm{P}_{3 / 2}$ & $4.78 \pm 0.18 \mathrm{~s}[17]$ \\
$\mathrm{Te}^{-}$ & $5 \mathrm{p}^{52} \mathrm{P}_{1 / 2} \rightarrow 5 \mathrm{p}^{52} \mathrm{P}_{3 / 2}$ & $0.463 \pm 0.008 \mathrm{~s}[17]$ \\
$\mathrm{Ni}^{-}$ & $3 \mathrm{~d}^{9} 4 \mathrm{~s}^{2} \mathrm{D}_{3 / 2} \rightarrow 3 \mathrm{~d}^{9} 4 \mathrm{~s}^{2}{ }^{2} \mathrm{D}_{5 / 2}$ & $15.1 \pm 0.4 \mathrm{~s}[18]$ \\
$\mathrm{Pt}^{-}$ & $5 \mathrm{~d}^{10} 6 \mathrm{~s}^{2} \mathrm{~S}_{1 / 2} \rightarrow 5 \mathrm{~d}^{9} 6 \mathrm{~s}^{2}{ }^{-} \mathrm{D}_{5 / 2}$ & $2.54 \pm 10 \mathrm{~s}[19]$ \\
$\mathrm{Pt}^{-}$ & $5 \mathrm{~d}^{9} 6 \mathrm{~s}^{22} \mathrm{D}_{3 / 2} \rightarrow 5 \mathrm{~d}^{9} 6 \mathrm{~s}^{22} \mathrm{D}_{5 / 2}$ & $50-200 \mathrm{~ms}[19]$ \\
\hline
\end{tabular}

Table 1: Measured radiative lifetimes of long-lived excited states in atomic anions. These are important benchmarkers for theoretical methods treating electron correlation effects (for more details and recent theoretical results see e.g. Ref [22] and references therein).
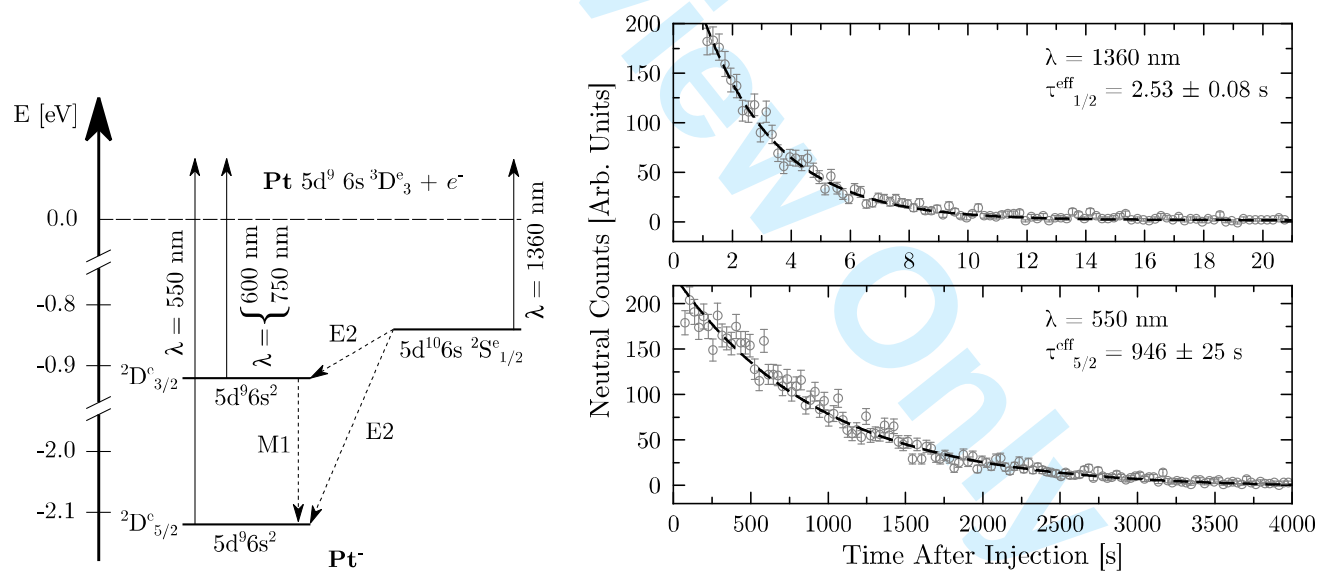

Figure 2: (Left) Energy level diagram showing the three bound states of $\mathrm{Pt}^{-}$. (Right) The $\mathrm{Pt}^{-}$decay curves when only one excited state is detached and when all three states are detached are shown (with different timescales) in the upper and lower panels, respectively. Reprinted figure with permission from Ref. [19]. Copyright 2016 by the American Physical Society.

In Table 1, we show radiative lifetimes of long-lived excited bound states in $\mathrm{S}^{-}, \mathrm{Se}^{-}, \mathrm{Te}^{-}$, $\mathrm{Ni}^{-}$, and $\mathrm{Pt}^{-}$that have been measured in DESIREE These results were obtained by probing the populations of the corresponding states at different times after injection in the symmetric DESIREE ion storage ring, as illustrated in the example in Fig. 2. The left part of this figure shows the energy level diagram for the three bound states of $\mathrm{Pt}^{-}$. 
The excitation energy of the $5 \mathrm{~d}^{9} 6 \mathrm{~s}^{2}{ }^{2} \mathrm{D}_{3 / 2}$ and $5 \mathrm{~d}^{10} 6 \mathrm{~s}^{2} \mathrm{~S}_{1 / 2}$ levels lie $1.20775(6) \mathrm{eV}$ [23] and $1.27567(161) \mathrm{eV}$ [24] above the $5 \mathrm{~d}^{9} 6 \mathrm{~s}^{2}{ }^{2} \mathrm{D}_{5 / 2}$ ground state, respectively. Electric dipole (E1) transitions from the excited states are forbidden due to the parity selection rule and the lowest order transitions between the ground and excited states of $\mathrm{Pt}^{-}$are M1 (magnetic dipole) and E2 (electric quadrupole) transitions. In the upper right panel of Fig. 2 we show the decay curve when using a laser photon wavelength of $1360 \mathrm{~nm}$ for detachment. Here, only the $5 \mathrm{~d}^{10} 6 \mathrm{~s}^{2} \mathrm{~S}_{1 / 2}$ state was photo-detached. In the lower right panel we show the corresponding curve for a laser photon wavelength of $550 \mathrm{~nm}$ where all three bound states were detached. By fitting the data in the upper and lower right panels of Figure 2 to a single exponential function and a sum of two exponential functions, respectively, we found that the effective lifetimes for the $5 \mathrm{~d}^{10} 6 \mathrm{~s}^{2} \mathrm{~S}_{1 / 2}$ state and the ground state were $2.53 \pm 0.08 \mathrm{~s}$ and $946 \pm 25 \mathrm{~s}$. The latter corresponds to the ionbeam storage lifetime and was used to correct the intrinsic lifetime for the $5 \mathrm{~d}^{10} 6 \mathrm{~s}^{2} \mathrm{~S}_{1 / 2}$ state, which was then found to be $2.54 \pm 0.10 \mathrm{~s}$. This correction is made for ion beam losses due to residual gas collisions and imperfections in the ion storage conditions, which are likely to affect the excited and ground state ions similarly (for details see Ref. [19]). This is an example of a lifetime for an excited state of a different electron configuration from that of the ground state. In contrast to fine structure M1 transitions in e.g. $\mathrm{S}^{-}, \mathrm{Se}^{-}$, and $\mathrm{Te}^{-}$, such lifetimes may not be easily predicted from experimentally determined or precisely calculated transition energies [22].

\subsection{Rotational cooling of molecular anions}
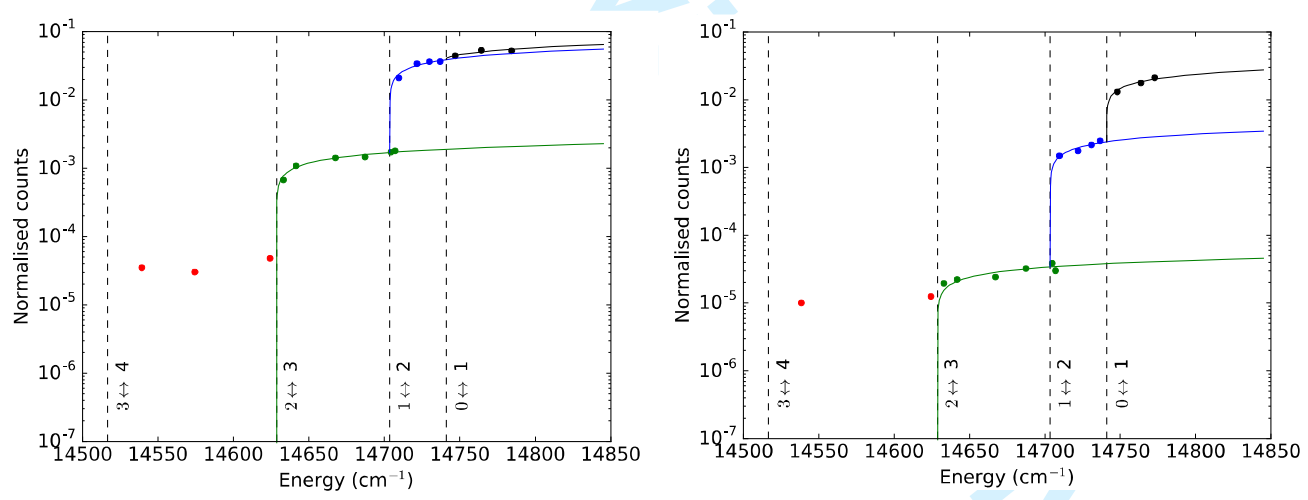

Figure 3: Normalized counts of neutrals as functions of the laser wavelength at $50 \mathrm{~s}$ (left panel) and $600 \mathrm{~s}$ (right panel) after ion beam injection.

Laser probing techniques may also be used to determine the rotational temperature of molecular ions that have been stored for long enough time to relax to their vibrational ground states. This was recently demonstrated in the CSR storage ring, where the rotational state populations for $\mathrm{CH}^{+}$were determined by monitoring the photodissociation yields as functions of the laser wavelength [20]. They [20] found that the rotational temperature of $\mathrm{CH}^{+}$reduces from more than $1000 \mathrm{~K}$ at the time of ion beam injection to about $20 \mathrm{~K}$ after storage for 240 seconds. We have used a similar technique where we photodetach vibrationally cold $\mathrm{OH}^{-}$ions that are stored at $10 \mathrm{keV}$ in one of the DESIREE storage rings. In Fig. 3, we show preliminary results for the yields of neutrals as functions of the laser photon energy in $\mathrm{cm}^{-1}$. The results for $\mathrm{OH}^{-}$stored for $50 \mathrm{~s}$ and $600 \mathrm{~s}$ are shown in the left and right panels, respectively. We observe energy 
windows with distinct thresholds (vertical dashed lines) for detachment of rotational states $\mathrm{J} \geq 0$ (black points), $\mathrm{J} \geq 1$ (blue points), and $\mathrm{J} \geq 2$ (green points). There is a significant increase in the relative population of the ground state $(\mathrm{J}=0)$ in the right panel compared to that shown in the left panel of Fig. 3. This suggests that the ions become rotationally colder as the storage time increases. In order to determine the relative populations and the related ion temperature as a function of storage time, we follow Ricco et al [25] and fit our data to the following expression for the photodetachment cross section

$$
\sigma(h v) \propto \sum_{J} P(J) I_{J}\left(h v-\varepsilon_{J}\right)^{p}
$$

where $\mathrm{P}(\mathrm{J}), \mathrm{I}_{\mathrm{J}}$, and $\varepsilon_{\mathrm{J}}$ are the population, the branching ratio, and the threshold energy of level $\mathrm{J}$, respectively. The exponent $\mathrm{p}$ describes the energy dependence of the cross section and is set to $\mathrm{p}=0.28$. The fits are shown as solid lines in Fig. 3 .

\begin{tabular}{|ccccccc|}
\hline Time $(\mathrm{s})$ & 50 & 100 & 150 & 300 & 600 & 900 \\
\hline $\mathrm{P}(0)$ & 0.34 & 0.71 & 0.81 & 0.92 & 0.95 & 0.95 \\
$\mathrm{P}(1)$ & 0.64 & 0.29 & 0.19 & 0.083 & 0.045 & 0.046 \\
$\mathrm{P}(2)$ & 0.026 & 0.0032 & 0.0013 & 0.00072 & 0.00057 & 0.00072 \\
\hline $\mathrm{T}(\mathrm{K})$ & - & 26.7 & 21.1 & 15.4 & 13.0 & 13.1 \\
\hline
\end{tabular}

Table 2: Population of rotational states, $\mathrm{P}(\mathrm{J})$, and rotational temperatures, $\mathrm{T}$, $\mathrm{of}^{-} \mathrm{OH}^{-}$at different ion beam storage times.

In Table 2 we show the results from the fits to six different ion beam storage times (50, $100,150,300,600$, and $900 \mathrm{~s}$ ). We see that the population of rotational states changes rather dramatically as a function of storage time until it reaches an equilibrium after 600 $\mathrm{s}$ where $95 \%$ of the ions are in the rotational ground state $(\mathrm{J}=0)$ and the remaining ions are mainly in $\mathrm{J}=1$. This corresponds to a rotational temperature of $13 \mathrm{~K}$, which shows that the ions then are in thermal equilibrium with the inner walls of the DESIREE vacuum chamber. By applying a second laser beam to selectively photo-detach rotationally excited ions with $\mathrm{J} \geq 1$ the effective temperature of the ions may be reduced even further. Recently, an ion beam with a $97 \%$ pure $J=0$ population has been prepared in this way in DESIREE.

\subsection{Cooling and decay of cluster anions}

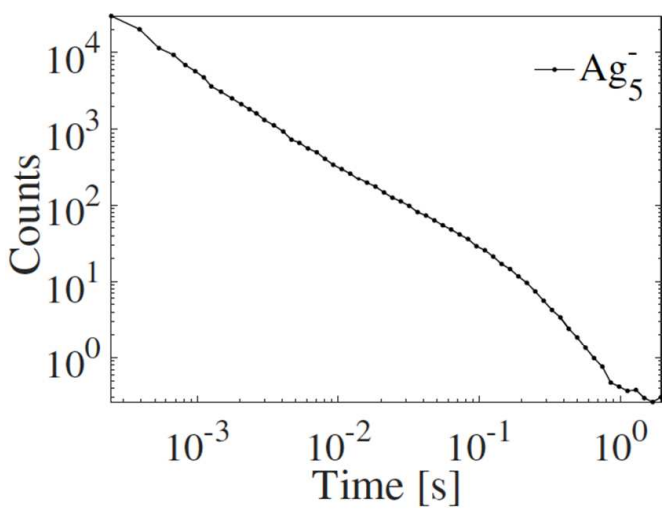


Figure 4: Rate of neutral particle emission from $\mathrm{Ag}_{5}{ }^{-}$clusters stored in one of the DESIREE ion-beam storage rings.

We have recently measured the cooling and decay for a large set of $\mathrm{C}_{\mathrm{n}}^{-}, \mathrm{C}_{\mathrm{n}} \mathrm{H}^{-}, \mathrm{Cu}_{\mathrm{n}}{ }^{-}[26]$, and $\mathrm{Ag}_{n}{ }^{-}$clusters in DESIREE. An example of preliminary results is shown in Fig. 4, where the number of emitted neutrals from decaying $\mathrm{Ag}_{5}{ }^{-}$anions is monitored as a function of ion storage time. The decay follows a power law for about $200 \mathrm{~ms}$, as expected for an ensemble of isolated systems with a broad internal energy distribution [2]. The deviation from the power law at longer timescales reveals some of the cooling dynamics, which could not be observed in the earlier storage ring experiments at room temperature [2]. There [2], collisions with the residual gas at much higher pressure than in DESIREE gave a much larger background (orders of magnitude higher) than what we have in DESIREE. Thus, we now have access to studies of cooling and decay processes in completely new time domains.

\section{Summary and conclusions}

In this short overview, we have demonstrated that it is possible to store anions for hours in one of the DESIREE storage rings. This has allowed us to measure the lifetimes of slowly decaying metastable excited states of atomic anions and to follow cluster cooling processes on orders of magnitude longer time scales than what has been possible before. By studying the rotational cooling of $\mathrm{OH}^{-}$, we have shown that it is possible to prepare internally cold molecular anion beams in DESIREE. This opens up for merged beams experiments under true interstellar conditions and a new tool for unraveling the full details of ion-ion reaction and ion cooling processes.

\section{Acknowledgements}

This work was supported by the Swedish Research Council (Contract No. 621-20123660). The author would like to acknowledge Henrik Cederquist and Henning Schmidt for their many useful comments on the manuscript, and the COST action CM1204 XUV/X-ray Light and Fast Ions for Ultrafast Chemistry (XLIC).

\section{References}

[1] J. B. Fenn et al, Science, vol. 246, p. 64, 1989.

[2] K. Hansen et al, Phys. Rev. Lett, vol. 87, p. 123401, 2001.

[3] G. Ito et al, Phys. Rev. Lett., vol. 112, p. 183001, 2014.

[4] S. B. Nielsen et al, Phys. Rev. Lett., vol. 87, p. 228102, 2001.

[5] S. B. Nielsen et al, J. Phys. B., vol. 37, p. R25, 2004.

[6] S. Martin et al, Phys. Rev. Lett, vol. 110, p. 063003, 2013.

[7] S. Tomita et al, J. Chem. Phys., vol. 124, p. 024310, 2006.

[8] S. P. Møller et al, Nucl. Instrum. Meth. A, vol. 394, p. 281, 1997.

[9] S. Jinno et al, Nucl. Instrum. Meth. A, vol. 532, p. 477, 2004. 
[10] J. Bernard et al, Rev. Sci. Instrum., vol. 79, p. 075109, 2008.

[11] R. D. Thomas et al, Rev. Sci. Instrum., vol. 82, p. 065112, 2011.

[12] H. T. Schmidt et al, Rev. Sci. Instrum., vol. 84, p. 055115, 2013.

[13] R. v. Hahn, Nucl. Instrum. Meth. B., vol. 269, p. 2871, 2011.

[14] Y. Nakano et al, J. Phys. Conf. Series, vol. 388, p. 142027, 2012.

[15] P. Reinhed et al, Phys. Rev. Lett, vol. 103, p. 213002, 2009.

[16] S. Menk et al, Phys. Rev. A, vol. 89, p. 022502, 2014.

[17] E. Bäckström et al, Phys. Rev. Lett., vol. 114, p. 143003, 2015.

[18] M. Kaminska et al, Phys. Rev. A, vol. 93, p. 012512, 2016.

[19] K. C. Chartkunchand et al, Phys. Rev. A, vol. 94, p. 032501, 2016.

[20] A. P. O'Connor et al, Phys. Rev. Lett., vol. 116, p. 113002, 2016.

[21] H. T. Schmidt, Physica Scripta, vol. T166, p. 014063, 2015.

[22] T. Brage and J. Grumer, J. Phys. B. At. Mol. Opt. Phys., vol. 50, p. 025001, 2017.

[23] J. Thøgersen et al, Phys. Rev. Lett., vol. 76, p. 2870, 1996.

[24] P. Andersson et al, Phys. Rev. A, vol. 79, p. 022502, 2009.

[25] O. Rico et al, Phys. Chem. Chem. Phys., vol. 15, p. 612, 2013.

[26] M. H. Stockett et al, Journal of Physics Conference Series, vol. 635, p. 072090, 2015. 